\title{
El discipulado de los laicos para el servicio integral en el mundo: un experimento misiológico evangélico a favor de las personas en situación de desplazamiento en Colombia"
}

\author{
Christopher Michael Hays ${ }^{* *}$
}

Recepción: 22 de agosto de 2018 • Aprobación: 27 de septiembre de 2018

\section{Resumen}

Este artículo describe el proyecto Fe y Desplazamiento para mostrar cómo una institución evangélica busca movilizar a la Iglesia a responder a los signos de los tiempos, específicamente con respecto al flagelo del desplazamiento forzoso, por medio de propiciar la evangelización integral y el crecimiento en la fe de laicos evangélicos, para que sean cocreadores y cogestores de la misión de la Iglesia. Describe el fundamento teórico del proyecto - una fusión de la misiología integral con la investigación-acción participación (IAP) - y brinda un resumen de la estructura del proyecto, con énfasis en la intervención pedagógico-pastoral que el proyecto ha elaborado. A lo largo del artículo, se indica cómo este acercamiento refleja varias de las prioridades de la Conferencia de Medellín.

Palabras clave: desplazamiento forzoso, investigación-acción participación, misión integral, Orlando Fals Borda, profesionales laicos.

* Artículo de investigación desarrollado en el marco del proyecto Fe y Desplazamiento, en la Fundación Universitaria Seminario Bíblico de Colombia, fue presentado en el Primer Congreso Internacional de Teología Latinoamericana y del Caribe: 50 años de Medellín: Iglesia y signos de los tiempos, desarrollado en la Universidad Santo Tomás entre el 16 y el 19 de octubre de 2018. Citar como: Hays, C. M. (2019). El discipulado de los laicos para el servicio integral en el mundo: un experimento misiológico evangélico a favor de las personas en situación de desplazamiento en Colombia. Albertus Magnus, X(1), 13-32. Doi: https://doi.org/10.153322/5005413.5108.

** Fundación Universitaria Seminario Bíblico, Colombia. Orcid: https://orcid.org/00000001-5050-4918. Correo electrónico: chays@unisbc.edu.co 


\title{
Discipling laity for integral service in the world: A missiological evangelical experiment in relation to internally displaced persons in Colombia
}

\begin{abstract}
This article describes the Faith and Displacement project in order to show how an evangelical institution seeks to mobilize the Church to respond to the signs of the times, especially in relation to forced displacement in Colombia, by means of fostering the integral evangelization and growth in faith of lay evangelicals, so that they become cocreators and coleaders in the mission of the Church. It describes the theoretical foundation of the project -a fusion between integral missiology and Participatory Action Research- and offers a summary of the project structure, emphasizing the pastoral-pedagogical intervention which the project has creatEd. Throughout the article, an account is given how this approach reflects various priorities of the Medellín conference.

Keywords: forced displacement, participatory action research, integral mission, Orlando Fals Borda, lay professionals.

\section{O discipulado dos leigos para o serviço integral no Colômbia} mundo: uma experiência missiológica evangélica em favor de pessoas em situação de deslocamento na
\end{abstract}

\section{Resumo}

Este artigo descreve o projeto Fé e Deslocamento para mostrar como é que uma instituição evangélica procura mobilizar a Igreja para responder aos sinais dos tempos, especificamente no que diz respeito ao flagelo do deslocamento forçado, promovendo a evangelização integral e o crescimento da fé dos leigos evangélicos, para ser cocriadores e cogestores da missão da igreja. Descreve a base teórica do projeto-uma fusão da missiologia integral com pesquisa-ação participativa-e oferece uma visão geral da estrutura do projeto, com ênfase na intervenção pedagógicopastoral que o projeto elaborou. Ao longo do artigo, é indicado como é que essa abordagem reflete várias das prioridades da Conferência de Medellín.

Palabras-chave: deslocamento forçado, pesquisa-ação participativa, missão integral, Orlando Fals Borda, profissionais leigos. 


\section{Introducción}

A veces, en Colombia, la interpretación de los signos de los tiempos es un tema de complejidad enloquecedora, en especial con respecto al conflicto armado. Son sumamente multifacéticos los debates sobre el equilibrio entre justicia, perdón e impunidad. Es impresionante el conjunto de complejidades al tratar de tender puentes de reconciliación hacia grupos armados sin querer premiar el terrorismo. No es nada fácil equilibrar consideraciones de la paz, la justicia y el salir adelante sin olvidar el pasado. Pero existen algunos temas que sí deben generar un acuerdo bipartito y unánime, aun en una Colombia polarizada como la nuestra. Uno de estos temas es la importancia de fomentar la recuperación de los 7400000 colombianos que son víctimas del desplazamiento forzoso (Red Nacional de Información [RNI], 2018). Estas personas - más del 15 \% de la población nacional- a menudo viven en condiciones horrorosas; por ejemplo, en casos de desplazamientos de zonas rurales a zonas urbanas, hay una tasa del $85 \%$ de pobreza extrema (Carillo, 2009, p. 534). Así las cosas, en principio, todos debemos estar de acuerdo en que esta población se tiene que socorrer. La interpretación de los tiempos en esta relación no requiere dones ni socioexegéticos ni político-místicos, por lo menos al nivel básico. A dicho nivel, debe quedar claro que Dios llama a su Iglesia a responder con solidaridad, compasión y proactividad para apoyar la recuperación de las personas en situación de desplazamiento. Sin embargo, con el propósito de saber concretamente cómo la Iglesia debe organizar sus esfuerzos para optimizar la recuperación de las personas en situación de desplazamiento, sí se requiere de mayor sofisticación.

El presente autor es profesor de la Fundación Universitaria Seminario Bíblico de Colombia en la ciudad de Medellín (una institución evangélica) y director del proyecto Fe y Desplazamiento. Tal proyecto busca movilizar las Iglesias evangélicas colombianas para fomentar la recuperación holística de las personas en situación de desplazamiento en Colombia como parte de su misión integral. ${ }^{1}$ El presente artículo describe el proyecto Fe y Desplazamiento para mostrar cómo

1 Indudablemente, la Iglesia católica ha desempeñado un papel fundamental en sensibilizar al pueblo colombiano sobre esta crisis y en responder concretamente a ella (García, 2007, pp. 53-73). Sin embargo, queda muchísimo por hacer, de modo que la Iglesia católica no puede escatimar los aportes de otras comunidades cristianas que comparten no solo la misma lectura fundamental de los signos de los tiempos con respecto al desplazamiento forzoso, sino que también tienen más compromisos en común con la Iglesia de lo que popularmente se reconoce, incluso con respecto a diversas prioridades particulares enunciadas en el Documento de Medellín. 
la Iglesia evangélica está interpretando y respondiendo a los signos de los tiempos de manera que corresponde con muchas de las prioridades recalcadas en el Documento de Medellín, específicamente "Pastoral popular" y "Pastoral de élites".

En consecuencia, se expone cómo una institución evangélica actualmente busca movilizar a la Iglesia a responder a los signos de los tiempos, específicamente con respecto al flagelo del desplazamiento forzoso en Colombia, por medio de propiciar la evangelización integral y el crecimiento en la fe de los laicos evangélicos, para que ellos sean cocreadores y cogestores de la misión de la Iglesia en Colombia (Consejo Episcopal Latinoamericano [Celam], 1968, II.12). Comienza con un bosquejo breve del fundamento teórico del proyecto - una fusión de la misiología integral con la investigación-acción participación (IAP) - y después brinda un resumen de la estructura del proyecto y su secuencia, con énfasis particular en la intervención pedagógico-pastoral que el proyecto ha elaborado. En el transcurso de la disertación, se indicarán las diversas maneras en las que este acercamiento refleja varias de las prioridades resaltadas en la Conferencia de Medellín.

\section{Teoría del proyecto Fe y desplazamiento: una fusión de la misiología integral y la investigación-acción participativa}

Se inicia con el fundamento teórico del proyecto Fe y Desplazamiento. Para asegurar que los esfuerzos de las Iglesias, orientados a responder a la crisis del desplazamiento forzoso, abarquen las diversas facetas del sufrimiento vivido por las personas en situación de desplazamiento, el proyecto parte de la misiología (es decir, la teoría teológica de la misión de la Iglesia) más influyente del mundo evangélico latinoamericano: la misiología integral.

\subsection{La misiología integral}

A menudo, los teólogos católicos desconocen que, en en la década de 1960, en paralelo con la emergencia de la teología de la liberación (Escobar, 2012, pp. 6785; Padilla DeBorst, 2016, pp. 125-130), surgió en América Latina una teología evangélica que renunció al docetismo espiritualista de muchas formas del cristianismo del norte y resaltó que la búsqueda de la justicia y la misericordia era 
fundamental a la misión del pueblo de Dios. La misiología integral abogaba por una orientación hacia la misión cristiana que afirma el Reino de Dios sobre las dimensiones tanto espiritual como física de la humanidad. Esta funciona como un correctivo a las formas de la teología cristiana que dan prioridad a la espiritualidad, pero descuidan las necesidades materiales y sociales de las personas, así como a aquellas formas de teología cristiana que dan preferencia a las realidades sociales sobre las afirmaciones bíblicas de las realidades espirituales.

La misión integral no fue, en primer lugar, un movimiento académico. Creció a partir de una preocupación por las misiones y el evangelismo, e hizo hincapié en que las misiones contemporáneas deben abordar las necesidades físicas de las personas, así como sus necesidades espirituales. La conferencia de 1969, Congreso Latinoamericano de Evangelización (CLADE), celebrada en Bogotá, Colombia, solo un año después de la Conferencia del Celam en Medellín, dio voz a la conciencia latinoamericana de que la justicia para los pobres debía ser parte del Evangelio predicado por las Iglesias evangélicas.

Aunque este no es el contexto adecuado para dar una introducción sistemática a la teoría de la misión integral, ${ }^{2}$ cabe resaltar dos de los compromisos clave de la misión integral:

- Atención a las realidades sociales concretas de los contextos latinoamericanos (Padilla, 1986, pp. 16-26, 132-135, 164-179).

- Participación de los laicos en el ministerio de la Iglesia (Padilla, 1998b, pp. 405-435).

Padilla y los otros proponentes de la misión integral argumentan que la misión solo hace justicia a la enseñanza bíblica y a la situación concreta cuando es integral. En otras palabras, cuando es un cruce de fronteras (no solo geográficas sino culturales, raciales, económicas, sociales, políticas, etc.) con el propósito de transformar la vida humana en todas sus dimensiones, según el propósito de Dios, y de empoderar a hombres y mujeres para que disfruten de la vida plena que Dios hizo posible por medio de Jesucristo en el poder del Espíritu. (Padilla, 2000, p. 31)

En contraste con la tendencia evangélica de describir la misión de la Iglesia desde el punto de vista de la conversión espiritual de las personas, Padilla argumenta que, según un acercamiento verdaderamente bíblico a las misiones,

2 Algunos de los libros más importantes incluyen Padilla (1986, 1998a); para una introducción a la historia del movimiento, véase Padilla (2008). 
la misión de los discípulos no esaría limitada a hacer conversos con el fin de incrementar el número de los miembros de la Iglesia. Ella estaría dirigida, más bien, a hacer discípulos en el estilo de vida que reprudiciaría el ejemplo de Jesús: un ejemplo del amor incondicional hacia Dios y al prójimo, del servicio humilde y la solidaridad con el pobre (2004, p. 31).

Por esta razón, partidarios de este movimiento resaltan el uso del adjetivo "integral" para describir su teoría de la misión de la Iglesia. Para ellos, una Iglesia que en su misión no atiende a las necesidades concretas en los contextos reales de América Latina no es una misión cristiana digna del nombre. Tal como la misiología integral rechaza cualquier división entre los aspectos espirituales y materiales de la misión de la Iglesia, así también no tolera ninguna bifurcación eclesial entre los pastores (quienes supuestamente realizan el ministerio de la Iglesia) y los laicos (quienes a menudo solo reciben el ministerio de la Iglesia) (cf. Padilla DeBorst, 2016, pp. 67-69). Al contrario, Padilla asevera que

la práctica de la misión integral asume que la Iglesia y cada uno de sus miembros darán prioridad absoluta al seguimiento de Jesús respecto de un estilo de vida misionera: un modo de vida modelado en Jesús con el propósito de dar testimonio, con la palabra y la acción, a Jesucristo el Señor (2004, p. 34)

La implementación de esta misión no depende primariamente de "profesionales" que son teológica y académicamente capacitados. Al contrario, Padilla argumenta, “la misión integral demanda la 'desclericalización' de los ministros y la 'laicización' del clero. En otras palabras, requiere el reconocimiento de la naturaleza apostólica de toda la Iglesia" (2004, p. 45).

Cabe notar que lo que estos teólogos evangélicos describen como la "misión integral de la Iglesia", en muchos sentidos equivale a lo que la Conferencia de Medellín entiende por "evangelización”. El Documento de Medellín (Celam, 1968), por un lado, reconoce que la evangelización no se limita al bautismo del cristiano, sino que incorpora su formación para enfrentar los retos y las injusticias históricas y concretas del mundo real. Por otro lado, reconoce que los laicos también comparten una vocación apostólica a participar en esta evangelización del mundo.

La evangelización debe orientarse hacia la formación de una fe personal, adulta, interiormente formada, operante y constantemente confrontada con los desafíos de la vida actual en esta fase de transición 
[...] Esta evangelización debe estar en relación con "los signos de los tiempos". No puede ser atemporal ni ahistórica. (Celam, 1968, II.13)

El mismo documento afirma la importancia de comunicar a los laicos élites "que son apóstoles de su propio ambiente" (Celam, 1968, III.14).

Según esta concepción de la misión integral de la Iglesia, el proyecto Fe y Desplazamiento procura enfrentar la crisis del desplazamiento forzoso de manera holística, precisamente, como una tarea imperativa para la Iglesia evangélica colombiana. Pero como se indicó al comienzo de este artículo, el reconocimiento de la necesidad de responder desde la Iglesia al flagelo del desplazamiento es más sencillo que lo que es el discernimiento de precisamente cómo responder a las diversas y complejas facetas de la vivencia y recuperación del desplazamiento forzoso. Así, para entender mejor las ramificaciones del desplazamiento forzoso y así construir intervenciones eclesiales que responden a las distintas facetas del desplazamiento, el proyecto Fe y Desplazamiento decidió combinar la teoría de la misión integral con la IAP. Es esta fusión de una teoría teológica evangélica con un método de investigación sociocientífico que marca el proyecto actual como académicamente innovador, y no solo una re-aplicación de una perspectiva misiológica ya popular en el gremio evangélico.

\subsection{La investigación-acción participativa}

En términos demasiado resumidos, la IAP es un método investigativo sociocientífico inicialmente derivado del método de investigación-acción, el cual fue elaborado en la década de 1940 en los Estados Unidos por el psicólogo Kurt Lewin (Lewin, 1946, pp. 34-46), pero que cogió nueva vida y se desarrolló de gran manera cuando llamó la atención de los académicos latinoamericanos. El momento clave de su contextualización fue el Simposio Internacional de Cartagena en 1977, en el que se añadió la palabra participativa a investigación-acción (Fals Borda, 1999, p. 84; Leal, 2009, p. 23). Las dos líneas investigativas más prominentes de la IAP en América Latina durante las últimas décadas del siglo XX eran la de la sociología (ejemplificado por Orlando Fals Borda; cf. Fals Borda, 1997) y la de la pedagogía (ejemplificado por Paulo Freire; cf. Freire, 2000).

Los cuatro rasgos clave de la metodología son:

- La necesidad fundamental de una acción, una intervención, en la IAP.

- La naturaleza cíclica de la IAP.

- La necesidad irreducible de la participación comunitaria en la IAP.

- La devolución del conocimiento a la comunidad. 
En el primer aspecto, Fals Borda concluyó que una acción social, la praxis, era no simplemente una parte integral de la investigación, sino que también era la prueba ácida de cualquier teoría. “El último criterio de validez del conocimiento científico venía a ser, entonces la praxis entendida como unidad dialéctica formada por la teoría y la práctica" (1997, p. 28). Así, la IAP pretende estudiar fenómenos sociales con el propósito de, no solo de entenderlos, sino también de intervenir y transformar situaciones de injusticia y sufrimiento (Colmenares, 2012, p. 106). Sugiere que un estudio académico preliminar debe resultar en la elaboración de una intervención, la cual se implementa con el propósito de medir científicamente su impacto. Según este análisis, se busca revisar y reimplementar la intervención (Fals Borda, 1999, p. 78; Krause, 2002, p. 49; Walter, 2010, pp. 3-4).

Este comentario nos lleva al segundo criterio. En vez de pretender solucionar un tema por medio del primer acercamiento, los proyectos de la IAP habitualmente se dividen en cuatro fases que se repiten: a) diagnóstico preliminar, b) construcción de una intervención, c) ejecución de la intervención y d) reflexión sobre la intervención, con el propósito de revisarla y re-aplicarla (Balcazar, 2003, pp. 62-63; Colmenares, 2012, pp. 107-108; Fals Borda, 1999, pp. 24-25).

En tercer lugar, como indica la palabra participativa en investigación-acción participativa, el método se marca por un compromiso adicional de incorporar en todos los pasos posibles de la investigación a las personas de todos los sectores interesados en un fenómeno, en especial incluyendo a los no académicos y personas que han sido marginadas socialmente (Fals Borda, 1999, p. 74). Se pretende realizar un trabajo conjunto entre investigadores académicos y no académicos (estos se denominan "coinvestigadores") en busca de convergencias entre conocimientos populares y académicos (p. 75). Pero, además de fortalecer la investigación en sí, la participación de los coinvestigadores se considera vital para la concientización de los miembros de la comunidad sobre su capacidad de generar un cambio en sus propias circunstancias.

En cuarto lugar, y para finalizar, un compromiso cardenal de la IAP es la devolución del conocimiento adquirido en la investigación a los miembros de la comunidad en formas accesibles para sus perfiles educacionales, habitualmente no muy avanzados (Fals Borda, 1999, p. 79). Volveremos a este tema a continuación.

Las ventajas de la cooperación participativa son múltiples:

- Los coinvestigadores locales tienen conocimiento endógeno y matizado de sus propias circunstancias.

- La participación de coinvestigadores locales fomenta la cooperación proactiva de la comunidad en la investigación. 
- Todo esto genera un compromiso mayor de la comunidad en la intervención propuesta, el cual fortalece la probabilidad del éxito de la intervención (cf. Cornwall \& Jewkes, 1995, p. 1668; Von Unger, 2012, párr. 9).

Por razón de su rigor académico y su aporte social, la IAP ha llegado a ser una de las metodologías sociocientíficas más populares de América Latina (aun mientras perdió algo de su popularidad en la región del norte atlántico durante las décadas de 1980 y 1990) (Krause, 2002, p. 41). Por tal razón, representa un método sociocientífico prometedor y lo suficientemente contextualizado como para analizar e intervenir en la crisis del desplazamiento colombiano.

Volviendo a la evangelización, la teoría del proyecto Fe y Desplazamiento es que la misiología integral se puede fortalecer por medio de incorporar muchos elementos de la IAP. Se propone que estas dos teorías se pueden volver aliadas, dados sus compromisos comunes con la participación de los "no expertos" y su entrega a enfrentar concretas situaciones de injusticia. Entonces, este proyecto se ha marcado de diversas maneras por la IAP, especialmente en el diseño de su secuencia de acción, en la estrategia de elaborar una intervención según la investigación, implementarla y medir su impacto, en la participación de los coinvestigadores y en el diseño de sus investigaciones de campo (sin mencionar el esfuerzo central para devolver la información a la comunidad en una forma que le sea accesible y apropiada). Para iluminar algo de cómo este proyecto de misiología integral se ha construido bajo la influencia de la IAP y de manera que incorpora otros compromisos de la Conferencia de Medellín, brevemente se bosquejará la estructura y secuencia del proyecto.

\section{Estructura del proyecto}

\subsection{Equipos}

En reconocimiento del hecho de que el desplazamiento forzoso es una experiencia multifacética, el proyecto Fe y Desplazamiento está compuesto de seis equipos de investigación: misiología, economía, sociología, psicología, pedagogía e interacción con el sector público. El equipo de misiología es responsable de elaborar la justificación para la macroteoría del proyecto y poner los fundamentos teológicos para la cooperación entre la academia, las Iglesias y las comunidades en situación de desplazamiento forzoso. Por encima del cimiento teórico puesto por el equipo de misiología, trabajan los otros cinco equipos del proyecto. Estos cinco equipos se enfocan en distintos temas desde las ciencias sociales, y buscan 
combinar reflexiones teológicas y no teológicas, por razones que se explicarán a continuación. Cada uno de estos equipos está compuesto de una combinación de teólogos y científicos sociales, además de académicos y profesionales.

\subsection{Comunidades piloto y coinvestigadores}

Sin embargo, los académicos y profesionales no son los únicos agentes en el proyecto. Como se explicó, uno de los compromisos esenciales de la IAP es que las poblaciones de estudio no solo sirven como objetos de investigación, sino que se deben considerar cosujetos de la investigación, especialmente porque cuentan con pericia y experiencia no disponibles al investigador académico. Así, este proyecto ha buscado aprender de y cooperar directamente con seis comunidades en situación de desplazamiento alrededor de Colombia (las cuales se denominan "comunidades piloto"). ${ }^{3}$

En cada comunidad se nombraron coinvestigadores. Idealmente se identificó uno que es líder de una Iglesia u organización basada en la fe, y otro que es o fue miembro de la comunidad desplazada. Los coinvestigadores no solo facilitan y guían nuestra interacción con las comunidades, sino que también contribuyen con sus propias experiencias, pericias y perspectivas al proyecto. Ellos también fueron invitados a elaborar sus propios proyectos de investigación, en colaboración con uno de los seis equipos del proyecto, y estos proyectos enriquecieron en gran medida las reflexiones de los demás miembros de los equipos.

\subsection{Secuencia de investigación}

La secuencia del proyecto también es organizada según los principios de la IAP. Como se explicó, la IAP habitualmente promueve una secuencia de investigación que comienza con a) análisis de un fenómeno o problema, b) elaboración de una

3 Las comunidades piloto son las siguientes: a) El Granizal, en la frontera de los municipios Bello y Medellín, es el segundo asentimiento más grande de las PSD en toda Colombia. b) La Granja, un asentimiento de personas en situación de desplazamiento (PSD) en los alrededores del municipio de Puerto Libertador, en el departamento de Córdoba. c) Santa Viviana y Santa Cecilia Alta, barrios en los márgenes de la ciudad de Bogotá. d) La Grandeza de Dios y Mataredonda, asentimientos de PSD de la tribu indígena colombiana nasa, cercanos al municipio de Piendamó, en el departamento de Cauca. e) Batata, un pueblo pequeño a $40 \mathrm{~km}$ del municipio de Tierralta, en el departamento de Córdoba, que ha sido el sitio de un conflicto prolongado, del cual han resultado múltiples desplazamientos forzosos, después de los cuales muchos miembros de la población voluntariamente retornaron a sus hogares. f) Nelson Mandela, originalmente un barrio de invasión creado por PSD en la ciudad de Cartagena. 
intervención diseñada para aminorar o solucionar el problema, c) implementación de la intervención y d) análisis y evaluación del impacto de la intervención (Balcazar, 2003, pp. 62-63; Colmenares, 2012, pp. 107-108; Fals Borda, 1997, pp. 22-24). Según este análisis, se busca profundizar en la investigación, ajustar la intervención, aplicarla de nuevo y medir otra vez su impacto, de una manera cíclica que teóricamente nunca tiene que finalizar. ${ }^{4}$

La investigación actual está estructurada en tres fases, de aproximadamente un año cada una. La primera fase (de julio de 2016 a junio de 2017) fue denominada fase de investigación. Esta fase se enfocó en una mezcla de investigación basada en la literatura académica e investigación de campo realizada en las seis comunidades piloto.

La segunda fase del proyecto (julio de 2017 a junio de 2018) se dedicó al desarrollo de una intervención educativo-misiológica, la cual incluye cartillas, currículos, videos, productos artísticos y recursos electrónicos para las Iglesias y las personas en situación de desplazamiento (PSD). Elaboramos estos bajo el liderazgo del equipo de pedagogía, que se ha dedicado especialmente al desarrollo de estrategias de pedagogía eficaz para PSD. Estos currículos y cartillas se lanzaron entre abril y julio de 2018, precisamente, en las mismas comunidades piloto, en las que se hizo la investigación de campo. Cabe aclarar que uno de los compromisos clave de la IAP es la devolución de la información recopilada en la investigación a las mismas comunidades de base, por esto es necesario emplear formas que cuadran con sus maneras tradicionales de aprender y sus perfiles educativos (Fals Borda, 1997, pp. 39-40; Fals Borda, 1999, p. 79; Kindon, Pain \& Kesby, 2009, p. 94). En este sentido, la decisión de priorizar las directrices del equipo de pedagogía en la elaboración de nuestros materiales educativos corresponde a los ideales de la IAP y al compromiso de la misión integral con la movilización de los laicos en la obra de la Iglesia.

La tercera y última fase del proyecto Fe y Desplazamiento (julio de 2018 a junio de 2019) fue dedicada al análisis del impacto de la intervención. Volvimos por tercera vez a las comunidades piloto, aproximadamente 6-8 meses después del lanzamiento de los currículos, para medir el impacto preliminar de las cartillas y los currículos, y estudiar cómo se podrían mejorar en el futuro. Aunque nuestra subvención se acaba en julio de 2019, actualmente estamos elaborando propuestas para revisar la intervención y reimplementarla, conforme a los ideales de la IAP.

4 La financiación actual del proyecto es limitada a tres años, de modo que solo se realizará un ciclo de IAP en este proyecto. 


\section{La intervención educativo-misiológica y la Conferencia de Medellín}

Antes de concluir, cabe profundizar más en la naturaleza de la intervención educativo-misiológica del proyecto, para resaltar cómo corresponde a varios de los énfasis de la Conferencia de Medellín.

\subsection{Pastoral de profesionales}

"Pastoral popular", al notar que la evangelización del pueblo sufría "la escasez de personal apostólico y la deficiente adaptación de las estructuras eclesiales" (Celam, 1968, I.1), subrayó la importancia de educar al laico "para ser cocreador y gestor con Dios de su destino" (II.12); en este sentido, coincide con el énfasis de la misión integral en la movilización del laicado en la misión de la Iglesia. Sin embargo, el enfoque de "Pastoral popular" era en los elementos transversales de la fe (p. ej., la proclamación de la Palabra de Dios y la santa eucaristía; II. 9), sin resaltar la diversidad personal y vocacional de los laicos "populares". En buena medida, "Pastoral popular" habla de los laicos como una masa humana indiferenciada. En contraste, "Pastoral de élites" se enfoca detalladamente en la diferenciación de distintos grupos profesionales, incluso artistas, médicos, abogados, educadores, ingenieros, militares, agrónomos, banqueros y empresarios (I.2), aunque cabe subrayar que hoy día estas son cada vez más profesiones no de las élites, sino de la creciente clase media colombiana. "Pastoral de élites" resalta que tales profesionales "son apóstoles de su propio ambiente" (II.14), y sugiere que "en la formación del clero es preciso prestar mayor atención a este tipo de pastoral especializado, preparando [...] asesores especializados para estos grupos" (II.16).

Hay mucho que celebrar en esta formulación, aun si el documento a veces parece cosificar en alguna medida a los laicos pobres mientras exalta la importancia de las élites. Sin embargo (y sin querer ser demasiado crítico), a cincuenta años de la publicación de estos documentos, pocos cleros han logrado elaborar una pastoral especializada que estimule a los laicos (sean élites o no) a ser cocreadores y gestores en el trabajo de Dios en su contexto.

Veinte años después del Congreso de Medellín, los líderes evangélicos colombianos se reunieron en la misma ciudad de la eterna primavera y emitieron su propia declaración de Medellín, parcialmente en reacción a la Conferencia del Celam de 1968. Estos líderes evangélicos colombianos afirmaron que el ser humano "fue creado como ser integral" (2. ․ parte, III.A.1), y se comprometieron a 
"desarrollar un discipulado que prepare al creyente para su servicio integral en el mundo" (2. ㅁ parte, IV.B.2), de manera que se compartió la prioridad expresada en "Pastoral de élites".

Sin embargo, después de varias décadas, e indudablemente por diversas razones, la realidad de ambas tradiciones cristianas sigue siendo marcada por la excesiva concentración de las responsabilidades y actividades de las Iglesias en las manos de los curas y pastores. Simultáneamente, los laicos habitualmente desempeñan papeles periféricos y no son discipulados para ser cocreadores y cogestores en el trabajo de Dios. Así las cosas, el proyecto Fe y Desplazamiento procuró elaborar su intervención educativo-misiológica de manera que pula y movilice a más laicos en la misión integral de la Iglesia a favor de las PSD.

Este tema se abordó primeramente en el currículo La misión integral de la Iglesia: cómo fortalecer o crear un ministerio a favor de personas en situación de desplazamiento, el cual es dirigido precisamente a líderes de la Iglesia para inculcar en ellos, por un lado, una comprensión de la misión integral de la Iglesia, y por otro, una eclesiología robusta que reconoce que el cuerpo de Cristo es uno pero tiene muchos miembros (1 Cor 12), de modo que es vital que el pastor reconozca que su responsabilidad no es realizar todo el ministerio de la Iglesia, sino discipular a sus ovejas para movilizarlas en la actualización de sus propias vocaciones dentro de la misión integral de la Iglesia (en este caso, a favor de las PSD) (Hays, Espitia y Villadiego, 2018a, pp. 46-54). Así, el proyecto Fe y Desplazamiento corresponde en buena medida a la recomendación de "Pastoral de élites": "en la formación del clero es preciso prestar mayor atención a este tipo de pastoral especializado [es decir, la pastoral de élites]" (Celam, 1968, II.16).

\subsection{La solidaridad y la participación}

El currículo también hace hincapié en el poder y el potencial de las mismas PSD (Hays et al., 2018a, pp. 63-86), puesto que su participación constructiva es esencial para el éxito de cualquier esfuerzo diseñado para fortalecer su recuperación. Esta verdad, a pesar de lo obvia que sea, a menudo se olvida en el afán de actuar a favor de los marginados, y si uno no tiene cuidado, en vez de acompañar al sufrido, se corre el riesgo de atropellarlo. Así, procuramos evitar cosificar a la persona desplazada o generar dinámicas de dependencia, precisamente, como resultado de una compasión expresada de manera exclusiva en formas de caridad y programas asistencialistas (sin negar el rol esencial de tales esfuerzos como parte de un portafolio de apoyos para la recuperación de la PSD). Con este fin, el 
currículo resalta el poder y el potencial de la persona desplazada, comunicando el punto por medio de una dramatización y de un estudio bíblico de 2 Cor 7-8, de videos que cuentan casos de superación por parte del esfuerzo de los mismos desplazados (Hays et al., 2018a, pp. 71-83) y de reflexiones devocionales sobre el Evangelio de Lucas (3, 8-11; 6, 20-24; 9, 57-58; 21, 1-4) (Hays, Espitia y Villadiego, 2018b, pp. 53-64). Además, desde el comienzo el currículo recalca la importancia de involucrar a miembros o líderes de la comunidad desplazada en este, para asegurar que sus voces se oigan y sus capacidades se vean desde el comienzo de las reflexiones de la Iglesia sobre cómo ministrar en su comunidad (Hays et al., 2018a).

En este sentido, desde este primer currículo, el proyecto Fe y Desplazamiento apoya el proceso de conocer y cooperar con la persona desplazada. "Debemos agudizar la conciencia del deber de solidaridad con los pobres, a que la caridad nos lleva. Esta solidaridad significa hacer nuestros sus problemas y sus luchas, saber hablar por ellos" (Celam, 1968, III.10). Afirmo completamente la importancia de abogar por la persona desplazada, pero quisiera sugerir que, primero, los que procuran practicar la solidaridad con el desplazado tienen que hablar con la persona desplazada, para después determinar juntos cuál es la mejor manera de actuar a favor de y en conjunto con la persona traspasada. Este currículo facilita el proceso de reflexión y actuación en conjunto, debido en buena medida a los énfasis tanto de la misiología integral como de la IAP. En la misma línea, "Pastoral popular" pronuncia que, "para la necesaria formación de estas comunidades [marginadas] se llame a una participación más activa en ellas a los religiosos, religiosas, catequistas especialmente preparados y apóstoles seglares" (Celam, 1968, III.14). Sin negar la veracidad de esta afirmación, cabe agregar que la formación de estas comunidades marginadas también puede realizarse a través de la movilización de los mismos miembros de las comunidades, aun si no cuentan con un catecismo profundo, en reconocimiento de su poder y dignidad, lo cual debe ser un elemento fundamental de trabajar "en solidaridad" con el pueblo.

Después de haber aclarado la importancia irreducible de la participación y el liderazgo proactivo de los laicos profesionales y de las mismas PSD en diversos aspectos de la misión de la Iglesia, los líderes de la Iglesia aplican un inventario de habilidades a los miembros de sus congregaciones para a) sistematizar los talentos profesionales, personales y comunitarios disponibles en los miembros de congregación (incluso los no muy conocidos al equipo pastoral) y b) sensibilizar a la gente a los aportes que ellos podrían hacer al ministerio con las PSD (Hays et al., 2018a, pp. 58-61). Según los resultados recopilados por el inventario de habilidades, los líderes colaboran con los miembros de su congregación y participantes de la comunidad desplazada en identificar cuáles de las otras "líneas" del proyecto ellos 
quisieran lanzar, conforme al capital humano de su congregación y las necesidades de la comunidad desplazada a su alrededor (Hays et al., 2018a, pp. 98-114).

\subsection{Las cinco líneas de formación y acción}

El proyecto cuenta con cinco líneas (más allá de la línea dedicada a la "Misión y el ministerio de la Iglesia"), cada una de las cuales corresponde a uno de los equipos investigativos del proyecto: economía, salud mental, enseñanza-aprendizaje, relaciones sociales y comunitarias, e interacción con el sector público. Cada línea del proyecto incluye recursos para capacitar laicos con pericias pertinentes a la línea y para poner sus habilidades y talentos (tanto profesionales como personales) al servicio de la misión de la Iglesia a favor de la comunidad desplazada. Al ser discipulados y capacitados con estos materiales, esos laicos pueden implementar los materiales de la línea diseñados para capacitar a miembros de la comunidad en situación de desplazamiento y acompañarlos en su recuperación en el aspecto de la vida abordado por la línea.

Por ejemplo, la línea de economía es diseñada para capacitar a personas con pericias relevantes a la generación de ingresos (es decir, empresarios, emprendedores, administradores de empresas, contadores, economistas, etc.), para que vean sus habilidades profesionales como parte de la misión de la Iglesia que pueden ser de mucho beneficio en el ministerio a favor de las personas en situación de desplazamiento. Comienza con una cartilla, titulada El profesional cristiano y la recuperación económica de las personas en situación de desplazamiento (Hays, 2018), diseñada para la capacitación tanto teológica como práctica de estos laicos. La cartilla incorpora videos educativos, actividades de reflexión y herramientas diagnósticas, y es la puerta de entrada a la línea.

Después de esta capacitación, estos profesionales pueden aprovechar diversos otros recursos y currículos que ellos pueden implementar con PSD que se quieran recuperar de la pobreza económica. Los materiales incluyen currículos sobre el desarrollo de microemprendimientos (Rehner et al., 2018), integración en el mercado laboral formal (Fajardo y Hays, 2018), principios cristianos éticos y teológicos pertinentes a temas económicos (Hays y Ramírez, 2018), e incorporan juegos, videos, dramatizaciones, estudios de casos, reflexiones devocionales, rompecabezas, etc. Así, la línea de economía brinda a los profesionales de las Iglesias capacitaciones, oportunidades y estructuras para desempeñar la misión integral de la Iglesia en favor de PSD y desde las habilidades y los talentos de los laicos mismos. 
Las otras cuatro líneas funcionan de manera similar, las cuales brindan capacitaciones y currículos a psicólogos, docentes, trabajadores sociales, abogados, funcionarios, entre otros, para que todos ellos puedan poner sus habilidades y talentos en práctica en la misión integral de la Iglesia a favor de las personas desplazadas (todos los materiales son gratuitos y están disponibles en la página web del proyecto, www.feydesplazamiento.org). En este sentido, el proyecto procura poner en práctica el tipo de "evangelización" que "Pastoral de élites" promueve, la cual "debe orientarse hacia la formación de una fe personal, adulta, interiormente formada, operante y constantemente confrontada con los desafíos de la vida actual", una evangelización "en relación con los 'signos de los tiempos'” (Celam, 1968, II 13).

\subsection{El rol del arte}

Finalmente, aunque la capacitación de los profesionales forma una parte central del acercamiento del proyecto Fe y Desplazamiento, también se tomó la decisión (gracias al impulso de unos estudiantes del seminario) de incorporar productos artísticos dentro del proyecto para sensibilizar a los miembros de las Iglesias a las realidades del desplazamiento forzoso y fortalecer su participación. Estos productos incluyen canciones, coro litúrgico, videos musicales, poemas e imágenes. La incorporación de estos productos artísticos en el proyecto también corresponde a una recomendación de "Pastoral de élites":

Teniendo en cuenta el papel que los artistas y hombres de letras están llamados a desempeñar en nuestro continente [...] como intérpretes naturales de sus angustias y esperanzas y generadores de valores autóctonos que configuran la imagen nacional [...] la Iglesia latinoamericana deberá dar, en su ámbito propio, el debido lugar a los artistas y hombres de letras, requiriendo su concurso para la expresión estética, de la palabra litúrgica, de la música sacra y de lugar de los lugares de culto. (Celam, 1968, III.17a, c)

Así, en varios sentidos, la intervención educativo-misiológica del proyecto Fe y Desplazamiento pone en práctica las recomendaciones pastorales de la Conferencia de Medellín. 


\section{Conclusiones: las correspondencias entre el Documento de Medellín y el proyecto Fe y Desplazamiento}

Ya hace cincuenta años, la Conferencia de Medellín reconocía que "la evangelización del continente experimenta serias dificultades, que se ven agravadas por [...] las migraciones internas, vencidas la escasez de personal apostólico y la deficiente adaptación de las estructuras eclesiales" (Celam, 1968, I.1). Hoy día, cuando la migración interna en forma del desplazamiento forzoso ha destruido las vidas de millones de colombianos, es más vital que nunca pensar de manera creativa y constructiva sobre cómo adaptar las estructuras eclesiales para responder a esa crisis y cómo capacitar a los laicos para superar la escasez de personal apostólico.

Por medio de presentar el proyecto de investigación institucional de la Fundación Universitaria Seminario Bíblico, se ha dado un ejemplo de cómo la teología evangélica latinoamericana ha querido responder a los signos de los tiempos. Efectivamente, el proyecto Fe y Desplazamiento procura movilizar más “apóstoles seglares" y adaptar las estructuras y los ministerios eclesiales para enfrentar la crisis de migración interna en Colombia.

Este artículo ha argumentado que el concepto evangélico de misión integral es efectivamente equivalente a lo que la Conferencia de Medellín entiende por "evangelización" y ha esbozado cómo el proyecto Fe y Desplazamiento ha elaborado una intervención educativo-misiológica para fomentar esa misión integral a favor de las personas en situación de desplazamiento. En particular, se ha subrayado cómo el proyecto resalta la pastoral de los profesionales, los grupos que el Documento de Medellín consideraba "élites", para hacerlos "apóstoles de su propio ambiente" (Celam, 1968, III.14; II.14). Pero el énfasis en la capacitación de los profesionales se complementa con la capacitación y el acompañamiento de las PSD, para que ellos participen también, no solo en su propia recuperación, sino también en la recuperación de sus prójimos. En este sentido, el proyecto busca contribuir a la evangelización y al crecimiento en la fe de los laicos y de los miembros "populares" de la Iglesia para que ellos lleguen a ser cada vez más cocreadores y cogestores con Dios en estos tiempos de conflicto y posconflicto en Colombia. 


\section{Referencias}

Balcazar, F. E. (2003). Investigación acción participativa (IAP): aspectos conceptuales y dificultades de implementación. Fundamentos en Humanidades, 4(1/2), 59-77.

Carrillo, A. C. (2009). Internal displacement in Colombia: Humanitarian, economic and social consequences in urban settings and current challenges. International Review of the Red Cross, 91(875), 527-546. https://doi.org/10.1017/S1816383109990427

Colmenares E., A. M. (2012). Investigación-acción participativa: una metodología integradora del conocimiento y la acción. Voces y Silencios: Revista Latinoamericana de Educación, 3(1), 102-115. Recuperado de https://revistas.uniandes.edu.co/doi/ pdf/10.18175/vys3.1.2012.07

Consejo Episcopal Latinoamericano. (1968). Documento de Medellín. Recuperado de http://www.diocese-braga.pt/catequese/sim/biblioteca/publicacoes_online/91/ medellin.pdf

Cornwall, A. \& Jewkes, R. (1995). What is participatory research? Social Science \& Medicine, 41(12), 1667-1676. https://doi.org/10.1016/0277-9536(95)00127-S

Escobar, S. (2012). Doing Theology on Christ's Road. En J. P. Greenman \& G. L. Green (Eds.), Global theology in evangelical perspective: Exploring the contextual nature of theology and mission (pp. 67-85). Downer's Grove, EE. UU.: Intervarsity.

Fajardo Sánchez, A. y Hays, C. M. (2018). Trabajos para la vida. Medellín, Colombia: Publicaciones SBC.

Fals Borda, O. (1997). El problema de cómo investigar la realidad para transformala por la praxis (7.. ed.). Bogotá, Colombia: Tercer Mundo.

Fals Borda, O. (1999). Orígenes universales y retos actuales de la IAP. Análisis Político, $38,71-88$.

Freire, P. (2000). Pedagogy of the oppressed (M. B. Ramos, trad.). Nueva York, Estados Unidos: Continuum.

García Durán, P. M. (2007). El papel de la Iglesia católica en la movilización por la paz en Colombia. En P. M. García Durán, O. C. Vélez, H. F. Henao y L. A. Castro Quiroga (Eds.), Creando un clima de reconciliación: escenarios para la verdad, la justicia y la paz (pp. 53-73). Bogotá, Colombia: Códice.

Hays, C. M. (2018). El profesional cristiano y la recuperación económica de las personas en situación de desplazamiento. Medellín, Colombia: Publicaciones SBC.

Hays, C. M. y Ramírez, H. L. (2018). La esperanza económica después del desplazamiento forzoso: manual del facilitador. Medellín, Colombia: Publicaciones SBC.

Hays, C. M., Espitia Zúñiga, I. y Villadiego Ramos, S. A. (2018a). La misión integral de la Iglesia: cómo fortalecer o crear un ministerio a favor de personas en situación de desplazamiento. Medellín, Colombia: Publicaciones SBC. 
Hays, C. M., Espitia Zúñiga, I. y Villadiego Ramos, S. A. (2018b). La misión integral de la Iglesia: cómo fortalecer o crear un ministerio a favor de personas en situación de desplazamiento: cuadernillo para participantes. Medellín, Colombia: Publicaciones SBC.

Kindon, S., Pain, R. \& Kesby, M. (2009). Participatory action research. En R. Kitchin \& N. Thrift (Eds.), International encyclopedia of human geography (pp. 90-95). Ámsterdam, Países Bajos: Elsevier.

Krause, M. (2002). Investigación-acción-participativa: una metodología para el desarrollo de la autoayuda, participación y empoderamiento. En J. Durston y F. Miranda (Comps.), Experiencias y metodología de la investigación participativa (pp.41-56). Santiago de Chile, Chile: Naciones Unidas. Recuperado de https:// repositorio.cepal.org/handle/11362/6023

Leal, E. (2009). La investigación acción participativa: un aporte al conocimiento y la transformación de Latinoamérica en permanente movimiento. Revista de Investigación, 67(33), 13-34. Recuperado de https://dialnet.unirioja.es/servlet/ articulo? codigo $=3220100$

Lewin, K. (1946). Action research and minority problems. Journal of Social Issues, 2(4), 34-46.

Padilla, C. R. (1986). Misión integral: ensayos sobre el Reino y la Iglesia. Buenos Aires: Argentina: Nueva Creación.

Padilla, C. R. (Ed.). (1998a). Bases bíblicas de la misión: perspectivas latinoamericanas. Buenos Aires, Argentina: Kairós.

Padilla, C. F. (1998b). Los "laicos" en la misión en el Nuevo Testamento. En C. R. Padilla (Ed.), Bases bíblicas de la misión: perspectivas latinoamericanas (pp. 405-435). Buenos Aires, Argentina: Kairós.

Padilla, C. R. (2000). Hacia una definición de la misión integral. En T. Yamamori y C. R. Padilla (Eds.), El proyecto de Dios y las necesidades humanas (pp. 19-33). Buenos Aires, Argentina: Kairós.

Padilla, C. R. (2004). Introduction: An ecclesiology for integral mission. En T. Yamamori y C. R. Padilla (Eds.), The local church, agent of transformation: An ecclesiology for integral mission (pp. 19-49). Buenos Aires, Argentina: Kairós.

Padilla, C. R. (2008). La trayectoria histórica de la misión integral. En T. Chester (Ed.), Justicia, misericordia y humildad: la misión integral y los pobres (pp. 55-80). Buenos Aires, Argentina: Kairós.

Padilla DeBorst, R. I. (2016). Integral mission formation in Abya Yala (Latin America): Astudy of the Centro de Estudios Teológicos Interdisciplinarios (1982-2002) and radical Evangélicos (Tesis doctoral, Boston University, Boston, EE. UU.). Recuperado de https://open.bu.edu/handle/2144/18651

Red Nacional de Información. (2018). Registro Único de Víctimas (RUV). Recuperado de https://rni.unidadvictimas.gov.co/RUV 
Rehner, S., Hays, C. M., Espitia Zúñiga, I., Fernández Rojas, J. P., Maestre Alvarado, E. E. y Villadiego Ramos, S. A. (2018). Desarrollo de la microempresa. Medellín, Colombia: Publicaciones SBC.

Unger, H. von (2012). Partizipative Gesundheitsforschung: Wer partizipiert woran? Forum: Qualitative Sozialforschung, 13(1), 1-29.

Walter, M. (2010). Participatory action research. En M. Walter (Ed.), Social research methods (2. ㄹ Ed.). Oxford, Reino Unido: Oxford University Press. 